\title{
Experimental Observation of Dirac Nodal Links in Centrosymmetric Semimetal TiB $_{2}$
}

\author{
Zhonghao Liu, ${ }^{1, *}$ Rui Lou, ${ }^{2, *}$ Pengjie Guo, ${ }^{2}$ Qi Wang, ${ }^{2}$ Shanshan Sun, ${ }^{2}$ Chenghe Li, ${ }^{2}$ Setti Thirupathaiah, \\ Alexander Fedorov, ${ }^{3,5}$ Dawei Shen, ${ }^{1}$ Kai Liu, ${ }^{2, \dagger}$ Hechang Lei, ${ }^{2, \$}$ and Shancai Wang ${ }^{2, \S}$ \\ ${ }^{1}$ State Key Laboratory of Functional Materials for Informatics and Center for Excellence in \\ Superconducting Electronics, SIMIT, Chinese Academy of Sciences, Shanghai 200050, China \\ ${ }^{2}$ Department of Physics and Beijing Key Laboratory of Opto-electronic Functional Materials \\ \& Micro-nano Devices, Renmin University of China, Beijing 100872, China \\ ${ }^{3}$ Institute for Solid State Research, IFW Dresden, D-01171 Dresden, Germany \\ ${ }^{4}$ Solid State and Structural Chemistry Unit, Indian Institute of Science, \\ Bangalore, Karnataka 560012, India \\ ${ }^{5}$ Department of Physics, St. Petersburg State University, St. Petersburg 198504, Russia
}

(Received 5 January 2018; revised manuscript received 7 July 2018; published 17 August 2018)

\begin{abstract}
The topological nodal-line semimetal state, serving as a fertile ground for various topological quantum phases, where a topological insulator, Dirac semimetal, or Weyl semimetal can be realized when the certain protecting symmetry is broken, has only been experimentally studied in very few materials. In contrast to discrete nodes, nodal lines with rich topological configurations can lead to more unusual transport phenomena. Utilizing angle-resolved photoemission spectroscopy and first-principles calculations, here, we provide compelling evidence of nodal-line fermions in centrosymmetric semimetal $\mathrm{TiB}_{2}$ with a negligible spin-orbit coupling effect. With the band crossings just below the Fermi energy, two groups of Dirac nodal rings are clearly observed without any interference from other bands, one surrounding the Brillouin zone (BZ) corner in the horizontal mirror plane $\sigma_{h}$ and the other surrounding the BZ center in the vertical mirror plane $\sigma_{v}$. The linear dispersions forming Dirac nodal rings are as wide as $2 \mathrm{eV}$. We further observe that the two groups of nodal rings link together along the $\Gamma-K$ direction, composing a nodal-link configuration. The simple electronic structure with Dirac nodal links mainly constituting the Fermi surfaces suggests $\mathrm{TiB}_{2}$ as a remarkable platform for studying and applying the novel physical properties related to nodal-line fermions.
\end{abstract}

DOI: 10.1103/PhysRevX.8.031044

Subject Areas: Condensed Matter Physics

\section{INTRODUCTION}

Topological materials with symmetry-protected nodes have recently attracted much attention in condensed matter physics. Perturbation that preserves a certain symmetry cannot remove the nodes by opening a full direct gap in these materials. When such nodes are close to the Fermi level $\left(E_{F}\right)$, the low-energy quasiparticle excitations are drastically different from the usual Schrödinger-type fermions. These nodes can be classified by their dimensionality $[1,2]$. The zero-dimensional

\footnotetext{
${ }^{*}$ These two authors contributed equally to this work.

Corresponding author.

kliu@ ruc.edu.cn

${ }^{*}$ Corresponding author.

hlei@ruc.edu.cn

${ }^{\S}$ Corresponding author. scw@ruc.edu.cn

Published by the American Physical Society under the terms of the Creative Commons Attribution 4.0 International license. Further distribution of this work must maintain attribution to the author(s) and the published article's title, journal citation, and DOI.
}

ones include Dirac points, Weyl points, threefold degenerate points and other higher-fold degenerate nodal points. With the four- or twofold degenerate Dirac or Weyl points, respectively, Dirac and Weyl semimetals have been theoretically predicted and experimentally confirmed [3-12]. More recently, threefold degenerate fermions, which conceptually lie between Dirac fermions and Weyl fermions, have been demonstrated to exist in the ZrTe family of compounds [13,14].

The nodal ring, nodal link, and nodal chain belong to the one-dimensional (1D) nodal-line systems whose nodes extend along 1D lines instead of discrete points in the threedimensional (3D) Brillouin zone (BZ) [2,15-19]. Protected by the combination of spatial-inversion symmetry and timereversal symmetry (the $P \cdot T$ symmetry) or certain crystalline symmetry $[20,21]$, the nodal line may either take the form of an extended line running across the $\mathrm{BZ}$, whose ends meet at the BZ boundary, or wind into a closed loop inside the BZ, or even form a chain consisting of several connected loops. A nontrivial Berry phase around the nodal lines would shift the Landau-level index by $1 / 2$ [17] and lead to drumhead surface states (SSs) [18,19].

Although band theory has predicted the existence of nodal-line fermions in some materials [19], only very 
few candidates have been experimentally studied by angleresolved photoemission spectroscopy (ARPES), i.e., $\mathrm{CaAgAs}, \mathrm{PbTaSe}_{2}$, and the ZrSiS family [20,22-26]. Very recently, two $\mathrm{AlB}_{2}$-type diborides $\mathrm{TiB}_{2}$ and $\mathrm{ZrB}_{2}$ have been proposed to possess nodal-line configurations coexisting with a pair of the triply degenerate nodal points (TNPs) with negligible spin-orbit coupling (SOC) [27,28]. The formation of the TNPs was well uncovered by calculations, but these nodes cannot be observed by ARPES with the crossings located above $E_{F}$. On the other hand, the nodal lines could be clearly observed with the crossing located below $E_{F}$. In this work, we unambiguously identify two groups of Dirac nodal rings in $\mathrm{TiB}_{2}$ by means of ARPES and first-principles calculations. The nodal rings embedded in the mirror plane $\sigma_{h}$ (the $\Gamma-K-M$ plane) around $K$ points compose one group and those in the mirror plane $\sigma_{v}$ (the $\Gamma-A-H$ plane) around $\Gamma$ point compose the other. Without interfering with other bands, the dispersions forming the nodal rings exhibit linearly in a wide energy range of approximately $2 \mathrm{eV}$. These two groups of nodal rings link together along the $\Gamma-K$ direction forming a nodal-link configuration, which goes beyond the isolated nodal line configuration in other systems. The compelling evidences of the nodal-line fermions existing just below $E_{F}$ and the nodal links mainly constituting the Fermi surfaces (FSs) provide an ideal system for further investigations and potential applications on transport phenomena of nodal lines.

\section{RESULTS AND DISCUSSION}

$\mathrm{TiB}_{2}$ has a simple $\mathrm{AlB}_{2}$-type centrosymmetric structure with the space group P6/ $\mathrm{mmm}$ (No. 191) [29]. As shown in Fig. 1(a), titanium and boron atoms lie in planar close-packed hexagonal layers alternately. Figure 1(b) shows the $\mathrm{x}$-ray diffraction (XRD) pattern of a $\mathrm{TiB}_{2}$ crystal, indicating that the measured surface is the $(00 l)$ plane. The mirrorlike (001) surface is illustrated in the inset of Fig. 1(b). In Fig. 1(c), the zero-field resistivity $\rho_{x x}$ of $\mathrm{TiB}_{2}$ exhibits a metallic behavior in the measured temperature range from 3 to $300 \mathrm{~K}$, and the magnetic field dependence of Hall resistivity $\rho_{x y}$ at $T=3 \mathrm{~K}$ is displayed in the inset of Fig. 1(c). Based on the nonlinear feature of $\rho_{x y}$ extending to high fields, electrons and holes are believed to coexist near $E_{F}$ in $\mathrm{TiB}_{2}$. Thus, we perform a quantitative analysis by fitting $\rho_{x y}$ with the two-carrier model (superimposed on the original data) [30]. The extracted carrier densities of $\mathrm{TiB}_{2}$ are $n_{e}=2.74(4) \times 10^{21} \mathrm{~cm}^{-3}$ and (a)

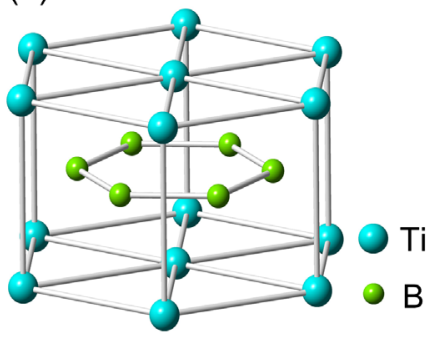

(d) (b)

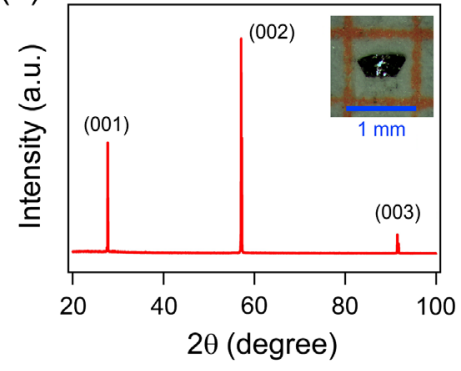

(e) (c)

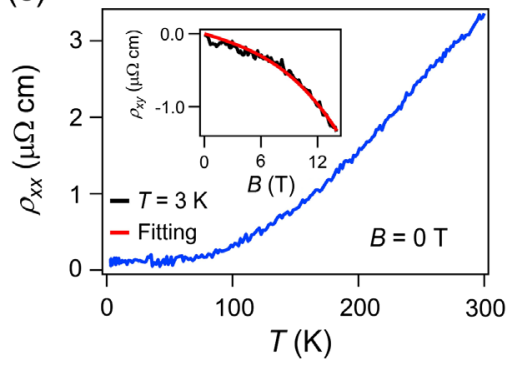

(f)
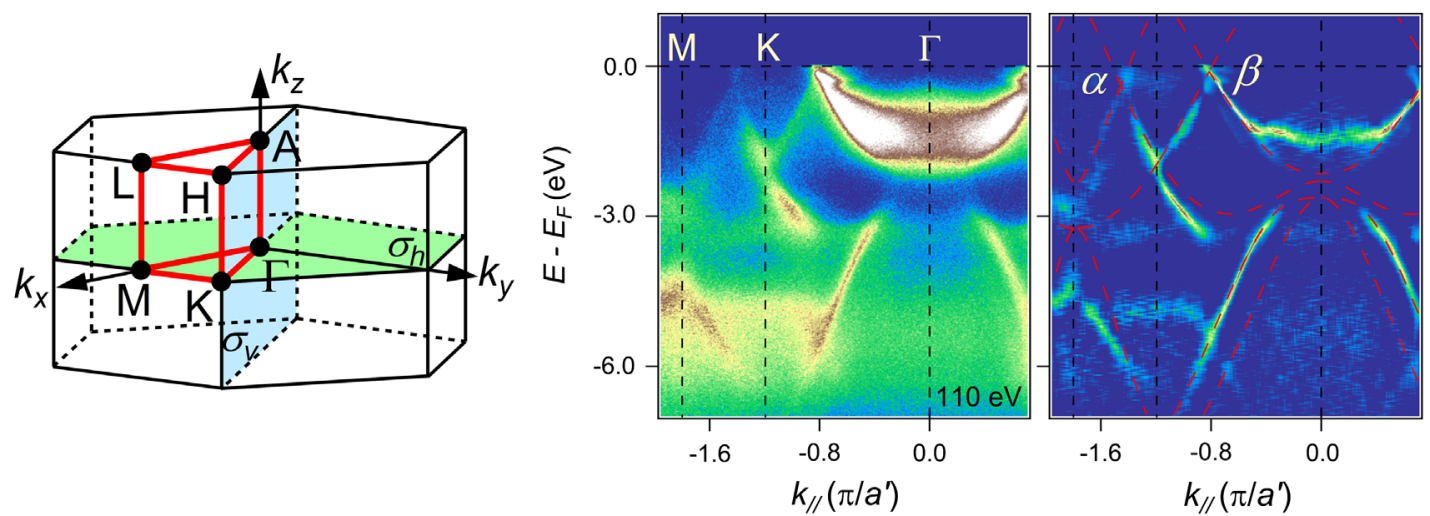

FIG. 1. Single-crystal and wide electronic structure of $\mathrm{TiB}_{2}$. (a) Crystal structure of $\mathrm{TiB}_{2}$ with space group P6/mmm (No. 191). (b) XRD pattern of a $\mathrm{TiB}_{2}$ single crystal. Inset: Picture of a $\mathrm{TiB}_{2}$ crystal against the 1-mm scale. (c) Temperature dependence of the resistivity $\rho_{x x}$ at $B=0 \mathrm{~T}$. Inset: Hall resistivity $\rho_{x y}$ (black curve) as a function of magnetic field $(B \|[001])$ at $T=3 \mathrm{~K}$, with the superimposed fitting result (red curve) using the two-carrier model. (d) 3D bulk BZ with marked high-symmetry points and two mirror planes, $\sigma_{h}\left(\Gamma-K-M\right.$ plane) and $\sigma_{v}(\Gamma-A-H$ plane). (e),(f) ARPES intensity plot and corresponding second derivative plot along the $\Gamma-K-M$ direction. $a^{\prime}=\sqrt{3} a / 2(a=3.0335 \AA)$. The red curves in (f) represent the calculated bulk bands with SOC. The band-crossing features along the $M-K$ and $K-\Gamma$ directions are denoted as $\alpha$ and $\beta$, respectively. 
$n_{h}=2.63(4) \times 10^{21} \mathrm{~cm}^{-3}$, respectively. They are much higher than those of Dirac semimetals like $\mathrm{Na}_{3} \mathrm{Bi}$ (approximately $10^{17} \mathrm{~cm}^{-3}$ [31]) and $\mathrm{Cd}_{3} \mathrm{As}_{2}$ (approximately $10^{18} \mathrm{~cm}^{-3}$ [32]) with discrete nodes, but comparable to that of nodal-line semimetals $\mathrm{ZrSi} M(M=S$, Se, and Te) $\left(n_{e}, n_{h} \sim 10^{20} \mathrm{~cm}^{-3}[33,34]\right)$. The concentrations in $\mathrm{TiB}_{2}$ estimated from first-principles calculations $\left(n_{e}, n_{h}=\right.$ $1.04,1.10 \times 10^{21} \mathrm{~cm}^{-3}$ ) also show good consistency with experimental results.

The bulk BZ and high-symmetry points are indicated in Fig. 1(d). The $\Gamma-K-M$ and $\Gamma-A-H$ planes are two mirror planes $\left(\sigma_{h}\right.$ and $\left.\sigma_{v}\right)$ of the $D_{6 h}$ group, respectively. Figures 1(e) and 1(f) show the ARPES intensity plot in a wide energy region along the $\Gamma-K-M$ direction and the corresponding second derivative plot, respectively. The calculated band dispersions have been superimposed on the second derivative plot, indicating the rather good agreement between the calculations and experimental data. Obviously, two linear band-crossing features denoted as $\alpha$ and $\beta$ can be identified in Fig. 1(f).

To comprehensively and accurately investigate the electronic structure of $\mathrm{TiB}_{2}$, we have scanned large portions of

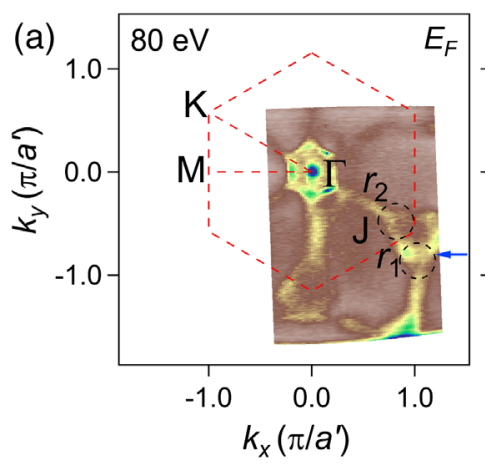

(c)

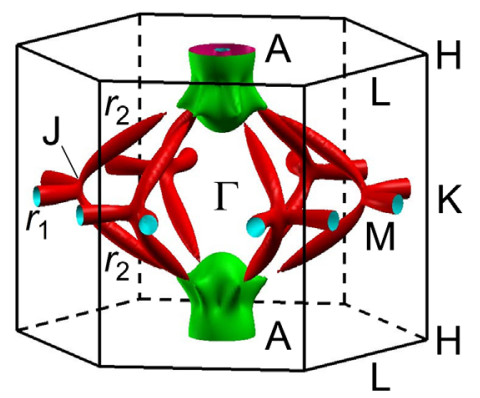

(d) $k$-space using various photon energies. The measured and calculated FSs are shown in Figs. 2(a)-2(d). Combining with the calculated band structure along high-symmetry lines, as displayed in Fig. 2(g), one can distinguish every FS in the whole 3D FS map of Fig. 2(c). Besides an electronlike and a holelike FS around the $A$ point in the $k_{z}=\pi$ plane, the overall FSs are mainly composed of the nodal rings, with the nodal rings $\left(r_{1}\right)$ embedded in the $\sigma_{h}$ plane around $K$ points linked with the ones $\left(r_{2}\right)$ in the $\sigma_{v}$ planes around the $\Gamma$ point at $J$ points along the $\Gamma$ - $K$ directions. Figure 2(d) shows the top view of the calculated 3D FSs, namely, the two-dimensional (2D) FSs projected on the (001) surface. With the FSs centered at the $A$ point, the $r_{1}$ nodal ring surrounding the $K$ point, the projection of the $r_{2}$ nodal ring around the $\Gamma$ point, and the $J$ nodal-link point all clearly resolved in Fig. 2(a), the experimental map taken with the photon energy of $80 \mathrm{eV}$ well reproduces the feature of the 2D projected FSs. Because the ARPES spectra reflect the electronic states integrated over a certain $k_{z}$ region of bulk $\mathrm{BZ}$, and because the electronic states at $k_{z}=0$ and $\pi$ have main contributions $[24,35]$, the $r_{1}$ nodal ring and the FSs centered at the $A$ point could be prominently distinguished in a certain range of
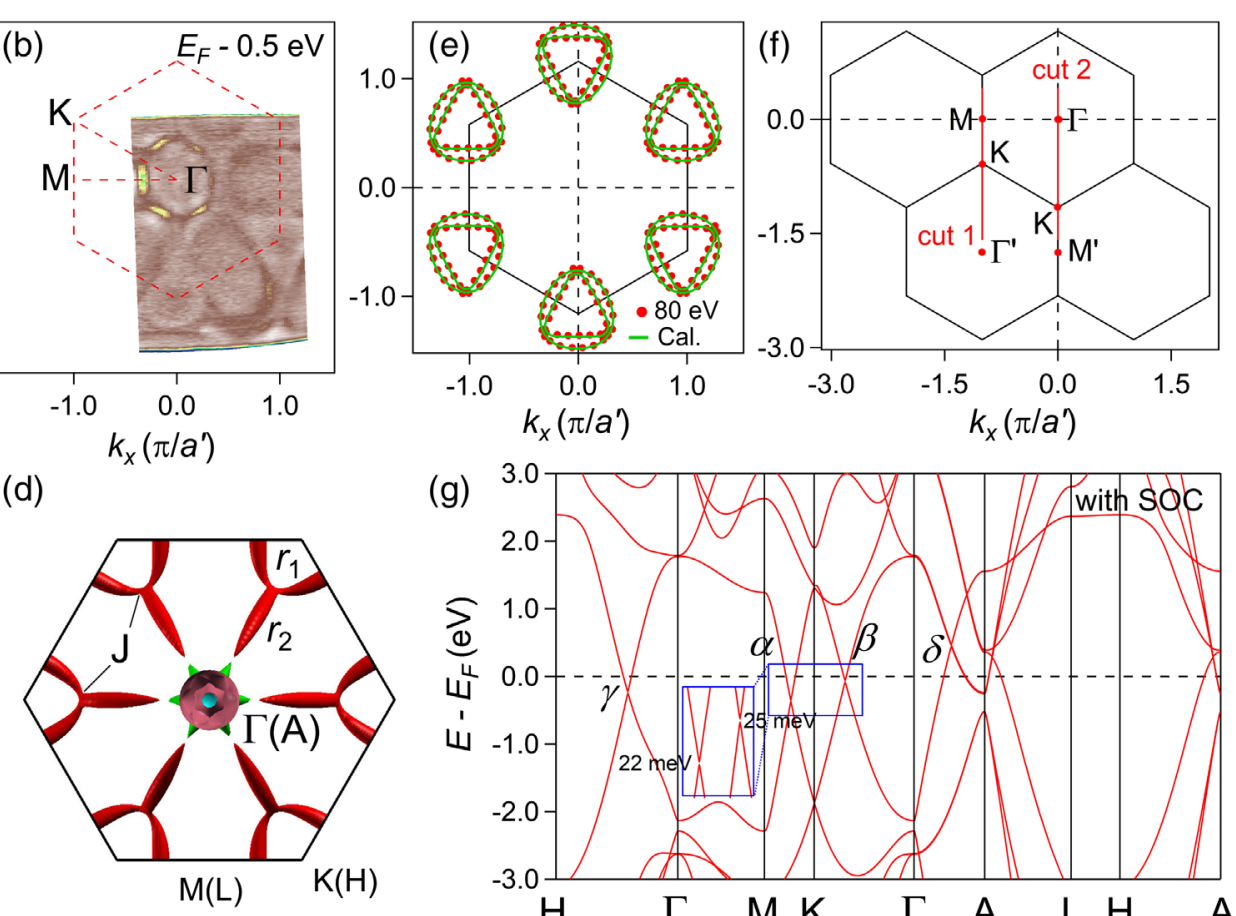

(g)

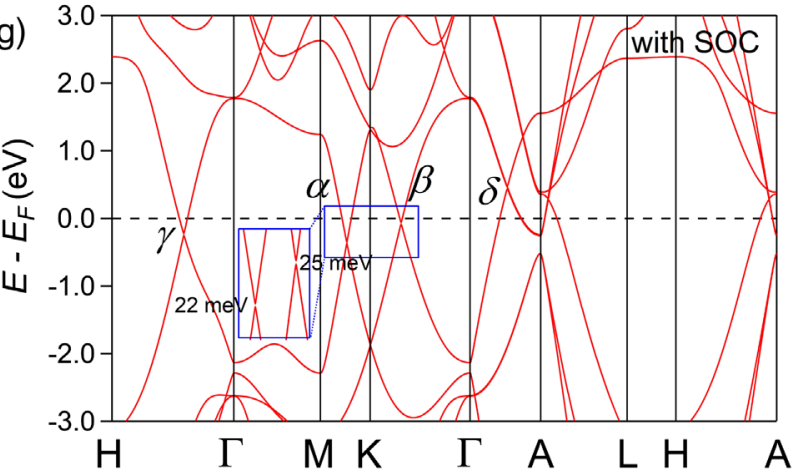

FIG. 2. FSs and calculated band structure of $\mathrm{TiB}_{2}$. (a),(b) Intensity plots at $E_{F}$ and $0.5 \mathrm{eV}$ below $E_{F}$ taken with $80-\mathrm{eV}$ photons. The red dashed lines indicate high-symmetry directions and the first BZ projected on the (001) surface. Two groups of Dirac nodal rings $r_{1}$ and $r_{2}$ [projected on the (001) surface] are resolved. The link points (denoted as $J$ points) of $r_{1}$ and $r_{2}$ are indicated by black dashed circles. (c),(d) Calculated bulk FSs in the 3D BZ and the top view of the FSs, respectively. The $r_{1}$ nodal ring surrounding the $K$ point is embedded in the $\Gamma-K-M$ plane. The $r_{2}$ nodal ring surrounding the $\Gamma$ point is embedded in the $\Gamma-A-H$ plane. (e) Comparison of the ARPES data and calculations for $r_{1}$. The green curves are calculated $r_{1}$ FSs. The red solid circles represent the experimental data, which are determined as follows: extracting the Fermi wave vectors from $1 / 3$ (marked by a blue arrow) of the $r_{1}$ nodal ring in (a); then, rotating it to a whole ring around the $K$ point; and last, symmetrizing to obtain other rings in the BZ. (f) Schematic 2D BZs with marked cuts 1 and 2 indicating the momentum locations of the measured bands in Fig. 3. (g) Calculated bulk band structure along high-symmetry lines including SOC. Four near- $E_{F}$ band-crossing features are denoted as $\alpha, \beta, \gamma$, and $\delta$, respectively. Inset: The enlarged view of $\alpha$ and $\beta$, small gaps of 22 and $25 \mathrm{meV}$ open at the nodes, respectively, when SOC is taken into consideration. 
photon energies. The banana-shaped $r_{1}$ nodal ring becomes a holelike pocket, when moving toward a high binding energy, as shown in Fig. 2(b).

In Fig. 2(g), one can find four band-crossing features near $E_{F}$ along the $M-K, K-\Gamma, H-\Gamma$, and $\Gamma-A$ directions, namely, $\alpha, \beta, \gamma$, and $\delta$, respectively. The nodes of the $\alpha$ and $\beta$ band-crossing features are located below $E_{F}$; thus, all of the $r_{1}$ nodal rings are resolved in the FS map. The nodes of the $\gamma$ and $\delta$ band-crossing features are below and above $E_{F}$, respectively, leading to the discontinuity of the $r_{2}$ nodal rings near the $A$ point, as illustrated in Figs. 2(c) and 2(d). Also, under the protection of the $C_{6 v}$ symmetry along the $\Gamma-A$ line, the node of the $\delta$ band-crossing feature is a TNP, which is formed by the crossing of a doubly degenerate band and a nondegenerate band. Because of time-reversal symmetry, a pair of the TNPs should locate at the two sides of the $\Gamma$ point. Further, the SOC introduces small gaps of $22,25,22$, and $33 \mathrm{meV}$ to the nodes of $\alpha, \beta, \gamma$, and $\delta$, respectively [see the calculated bulk band structure without SOC in Fig. S1 of the Supplemental Material (SM) [36] ]. The gap sizes are much less than those in CaAgAs (approximately $73 \mathrm{meV}$ ) [22] and $\mathrm{ZrSiTe}$ (approximately $60 \mathrm{meV}$ ) [26] and are comparable to those in $\mathrm{ZrSiS}$ and
ZrSiSe (approximately 25-35 meV) [20,26], indicating the weak SOC effect on the bulk electronic structure of $\mathrm{TiB}_{2}$. The $r_{2}$ nodal ring in the $\sigma_{v}$ plane formed by $\gamma$ and $\delta$ needs to be further demonstrated by the photon-energy-dependent studies, which will be discussed later. Here, we extract the Fermi wave vectors of $r_{1}$ according to the ARPES data and plot them in Fig. 2(e) with superimposed calculation contours. The size and shape of the calculated ones are very consistent with those determined by ARPES.

To quantitatively study the features of the $r_{1}$ nodal ring embedded in the $\sigma_{h}$ plane, we record the ARPES spectra near $E_{F}$ along the $M-K$ and $\Gamma-K$ directions, as indicated by cuts 1 and 2 in Fig. 2(f). The intensity plots and corresponding second derivative plots are presented in Figs. 3(a)-(d). The overall band structures agree well with the appended theoretical calculations. We also notice that some band dispersions near $\beta$ along the $\Gamma-K$ direction are not reproduced by bulk calculations. These bands most likely come from the contributions of bulk states in other $k_{z}$ planes due to the $k_{z}$ broadening effect $[24,35]$.

To avoid the interference with other bands, we use the photon energy of $110 \mathrm{eV}$, in principle corresponding to the $\Gamma-K-M$ plane, to record the intensity. As shown in Figs. 3(e)
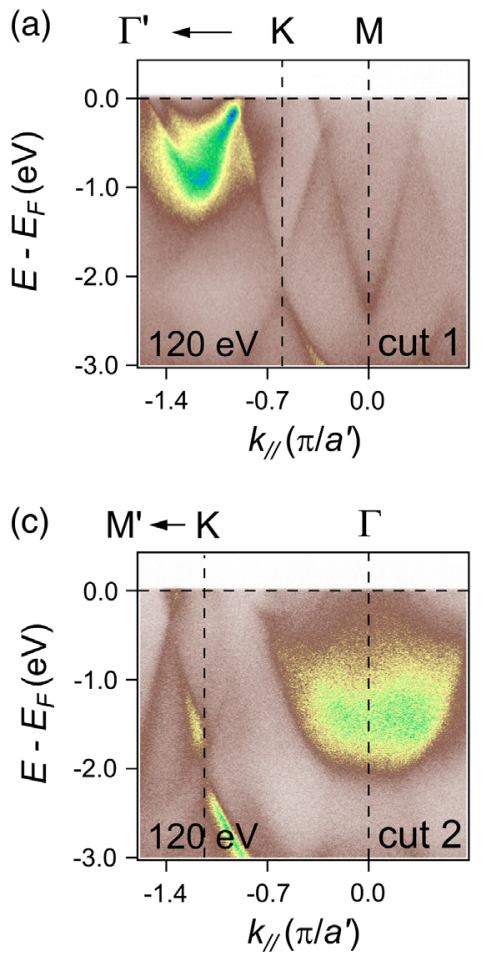

(b)

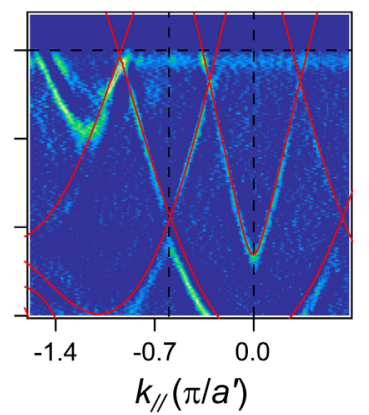

(d)

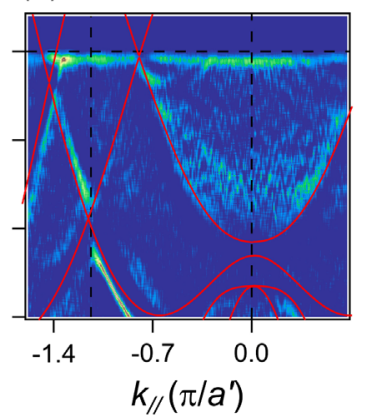

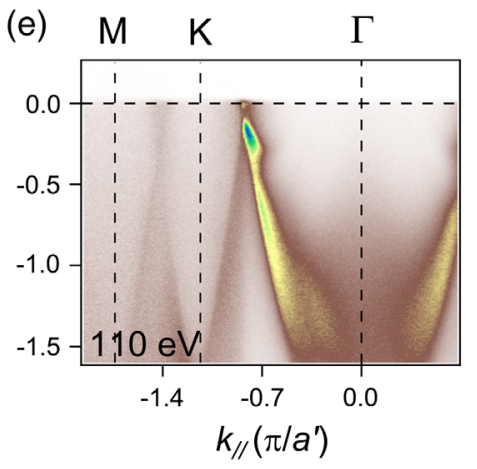

(f)

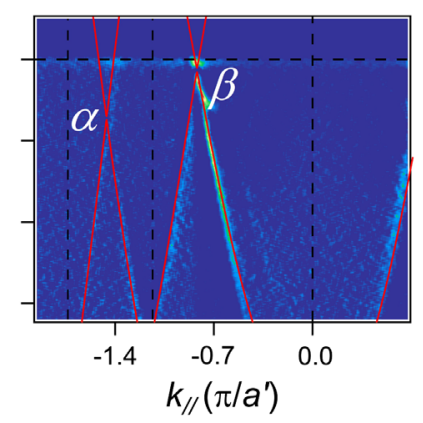

(g)

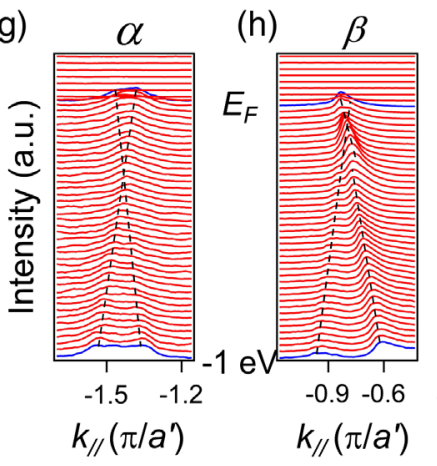

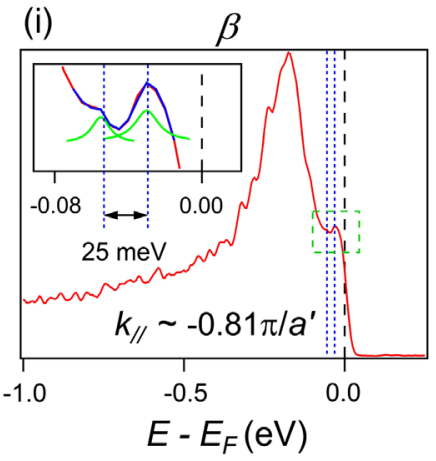

FIG. 3. Electronic structure of the $r_{1}$ nodal ring. (a),(b) Intensity plot and corresponding second derivative plot along the $M$ - $K$ direction [cut 1 in Fig. 2(f)] recorded at $h \nu=120 \mathrm{eV}\left(k_{z} \sim 0.4 \pi / c\right)$. (c),(d) Same as (a),(b), but along the $\Gamma$ - $K$ direction [cut 2 in Fig. 2(f)]. (e),(f) Same as (a),(b), but recorded along the $\Gamma-K-M$ direction with 110 -eV photons $\left(k_{z} \sim 0\right)$. The band-crossing features along the $M-K$ and $K-\Gamma$ directions, corresponding to the $r_{1}$ nodal ring surrounding the $K$ point, are denoted as $\alpha$ and $\beta$ in (f), respectively. The calculated bands with SOC are plotted on the second derivative plots in (b),(d), and (f). (g),(h) MDCs plots of $\alpha$ and $\beta$, respectively. The black dashed lines indicate the linear dispersions. (i) EDC taken at the center (approximately $-0.81 \pi / a^{\prime}$ ) of $\beta$. Inset: Zoomed in EDC near $E_{F}$ indicated by the green dashed rectangle. By fitting the EDC with two Lorentzian profiles, shown as green curves, an opening gap of $25 \mathrm{meV}$ can be determined. The blue curve is the superimposed fitting result. 

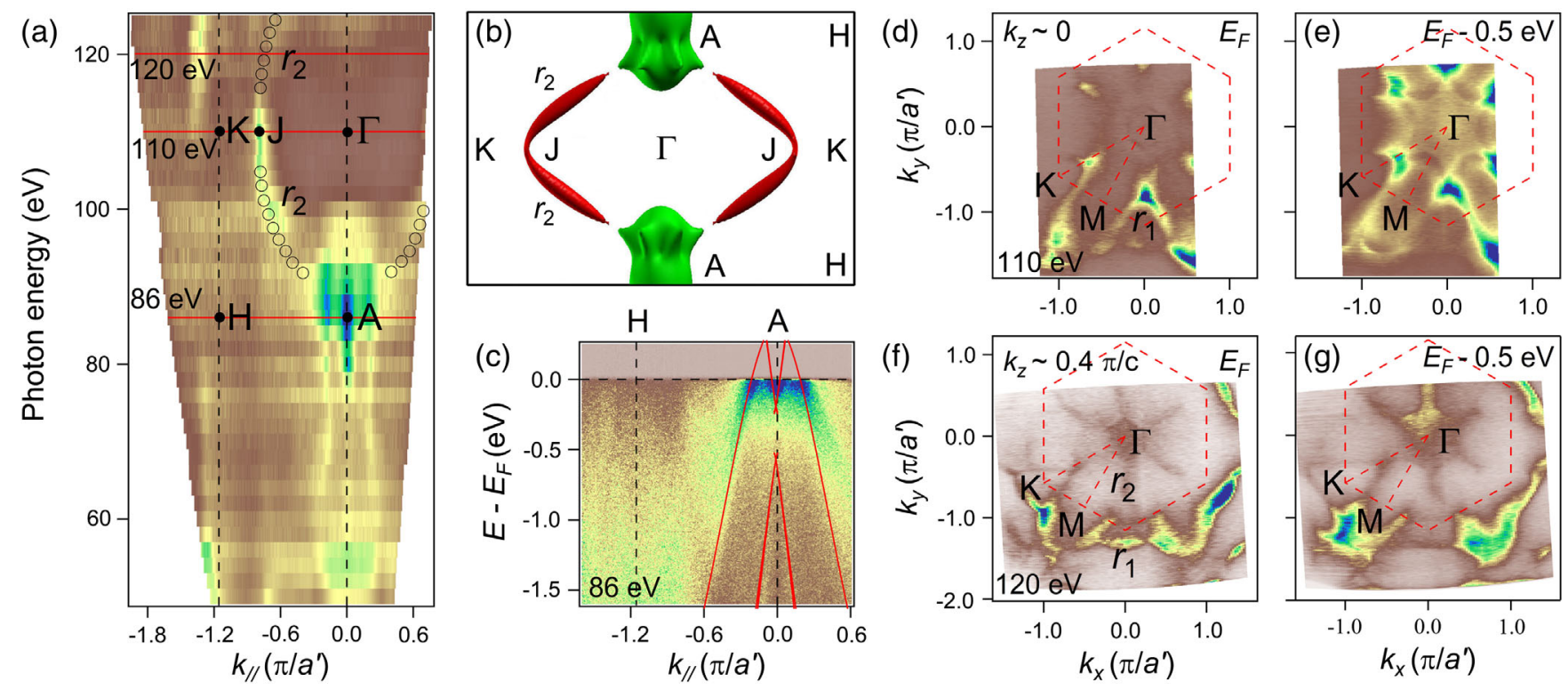

FIG. 4. Photon-energy-dependent dispersions of $\mathrm{TiB}_{2}$. (a) Intensity plot in the $h \nu-k_{/ /}$plane at $E_{F}$, with $k_{/ /}$oriented along the $\Gamma$ - $K$ $(A-H)$ direction. The high-symmetry points and nodal-link point are plotted. The $r_{2}$ nodal ring embedded in the $\Gamma$ - $A$ - $H$ plane surrounding the $\Gamma$ point is indicated by open circles. (b) Projections of calculated bulk FSs on the $\Gamma-A-H$ plane. (c) ARPES spectra taken along the $H$-A line with $86-\mathrm{eV}$ photons $\left(k_{z} \sim \pi\right)$. The appended red curves are bulk band calculations with SOC. (d, e) Constant-energy ARPES images at $E_{F}$ and $0.5 \mathrm{eV}$ below $E_{F}$ obtained by $110-\mathrm{eV}$ photons $\left(k_{z} \sim 0\right)$, respectively. (f), (g) Same as (d),(e), but taken with $120-\mathrm{eV}$ photons $\left(k_{z} \sim 0.4 \pi / c\right)$.

and 3(f), the $\alpha$ and $\beta$ band-crossing features are more outstandingly recognized. The corresponding momentum distribution curves (MDCs) in Figs. 3(g) and 3(h) unambiguously demonstrate the linear dispersions in a large energy range, and the nodes of $\alpha$ and $\beta$ are below $E_{F}$. The sizes of $\alpha$ and $\beta$ FSs along the $\Gamma-K$ direction are 0.11 and $0.07 \pi / a^{\prime}$, respectively. The result is in agreement with the data extracted from de Haas-van Alphen effect measurements [37]. To quantitatively determine the energy gaps at the nodes, we extract the energy distribution curve (EDC) at the center (about $-0.81 \pi / a^{\prime}$ ) of $\beta$, as illustrated in Fig. 3(i). By zooming in the near- $E_{F}$ area, one can clearly see two peaks in the inset of Fig. 3(i). We use two Lorentzian curves to fit this single EDC and superimpose the fitting result as a blue curve. The gap of approximately $25 \mathrm{meV}$ at the node of $\beta$ can be obtained, showing good consistency with theoretical calculation. Although the faint intensity due to the matrix element effect obstructs the determination of the band gap at $\alpha$, we can reasonably expect a small gap opening based on the high consistency between experiments and calculations.

As mentioned above, the $r_{2}$ nodal ring embedded in the $\sigma_{v}$ plane needs to be further demonstrated in the $k_{z}-k_{/ /}$ plane. We perform $k_{z}$-dependent measurements by varying the photon energy from 50 to $124 \mathrm{eV}$, covering more than one BZ along the $k_{z}$ direction. Figure 4(a) shows the intensity plot at $E_{F}$ as a function of photon energy and $k_{/ /}$, which is oriented along the $\Gamma-K(A-H)$ direction. One can see that the photoemission intensity is enhanced around the $A$ point $(86 \mathrm{eV})$ and suppressed around the $\Gamma$ point $(110 \mathrm{eV})$ along the $A-\Gamma$ direction, showing a periodic modulation along with photon energies. By comparing with the projections of calculated FSs on the $\Gamma-A-H$ plane in Fig. 4(b), the $r_{2}$ nodal rings passing through the $J$ nodallink point are traced out by open circles in Fig. 4(a). With the observation of both the (001) projection and the 3D character of the $r_{2}$ nodal ring in Figs. 2(a) and 4(a), respectively, the existence of nodal rings embedded in the $\sigma_{v}$ planes can be clearly proved. Thus, the nodal-link structure formed by the $r_{1}$ and $r_{2}$ nodal rings is confirmed.

Figure 4(c) shows the ARPES intensity plot along the $A-H$ line taken by the photon energy of $86 \mathrm{eV}$. As compared with the band structure along the $\Gamma-K$ direction in Fig. 3(e), an electronlike band and a holelike band cross $E_{F}$ around the $A$ point, while no band exists at the $\Gamma$ point. Moreover, the $\beta$ band-crossing feature along the $\Gamma-K$ direction disappears along the $A-H$ direction. These contrasts reveal the $3 \mathrm{D}$ nature of the band structure around the $A$ point and the embedding of the $r_{1}$ nodal ring in the $\sigma_{h}$ plane. Figures $4(\mathrm{~d})-4(\mathrm{~g})$ show the constant-energy maps in the $k_{x}-k_{y}$ plane, which are taken by the photon energies of $110 \mathrm{eV}\left(k_{z} \sim 0\right)$ and $120 \mathrm{eV}\left(k_{z} \sim 0.4 \pi / c\right)$, respectively. As illustrated in Fig. 4(f), the nodal-link configurations of $r_{1}$ and $r_{2}$ are also recognized like that in Fig. 2(a).

Besides the bulk evidence of the nodal line, the drumhead SSs appearing inside the projections of the nodal lines serve as the surface signature. As discussed above, small gaps will open at the nodes of $\alpha, \beta, \gamma$, and $\delta$, when including the SOC effect. Given the presence of the inversion center in $\mathrm{TiB}_{2}$, we calculate the product of the parities of the occupied states, which are all doubly degenerate due to the $P \cdot T$ symmetry, at 
the eight time-reversal invariant momenta with SOC [38]. The results are listed in Table S1 of the SM [36]. With a $Z_{2}$ index as $(0 ; 001)$, the $\mathrm{SSs}$ of $\mathrm{TiB}_{2}$ predicted in Refs. [27,28] are not topologically protected, and a small perturbation on the surface could hinder the observation of SSs in our experiments.

\section{CONCLUSION}

By systematically mapping out the 3D electronic structure both in experiment and theory, we unambiguously demonstrate the existence of nodal-line fermions in $\mathrm{TiB}_{2}$, hosting Dirac nodal links formed by two groups of nodal rings with a negligible SOC effect, under the protection of $P \cdot T$ symmetry and certain mirror reflection symmetries. The discovered features, i.e., the nodal-line fermions existing just below $E_{F}$ and the nodal links mainly constituting the FSs, must have a significant contribution to the low-energy excitations. The simple electronic structure composed of a large energy range of linearly dispersive bands offers a good platform for further studies into Dirac physics.

\section{ACKNOWLEDGMENTS}

We thank Zhong-Yi Lu for helpful discussions. The work was supported by the National Key R\&D Program of China (Grants No. 2016YFA0300504 and No. 2017YFA0302903), and the National Natural Science Foundation of China (Grants No. 11774421, No. 11574394, No. 11774423, No. 11774424, No. 11227902, and No. 11704394). R. L., K. L., and H. L. were supported by the Fundamental Research Funds for the Central Universities, and the Research Funds of Renmin University of China (RUC) (Grants No. 17XNH055, No. 14XNLQ03, No. 15XNLF06, and No. 15XNLQ07). Z. L. acknowledges Shanghai Sailing Program (Grant No. 17YF1422900). A.F. acknowledges the support of Saint Petersburg State University (Grant No. 15.61.202.2015).

\section{APPENDIX: MATERIALS AND METHODS}

\section{Sample synthesis}

Single crystals of $\mathrm{TiB}_{2}$ were grown by the Co flux method. The starting elements of $\mathrm{Ti}(99.995 \%)$, B $(99.99 \%)$, and Co $(99.99 \%)$ were put into an alumina crucible, with a molar ratio of $\mathrm{Ti}: \mathrm{B}: \mathrm{Co}=1: 2: 4$. The mixture was heated up to $1873 \mathrm{~K}$ in a high-purity argon atmosphere and then slowly cooled down to $1623 \mathrm{~K}$ at a rate of $4 \mathrm{~K} / \mathrm{h}$. The $\mathrm{TiB}_{2}$ single crystals were separated from the Co flux using a hot hydrochloric acid solution.

\section{ARPES measurements}

ARPES measurements were performed at the UE112 PGM-2 ARPES end station of BESSY using photon energies from 50 to $124 \mathrm{eV}$. The energy and angular resolutions were set to $15 \mathrm{meV}$ and $0.2^{\circ}$, respectively. Samples were cleaved in situ, yielding flat mirrorlike (001) surfaces. During the measurements, the temperature was kept at $40 \mathrm{~K}$ and the pressure was maintained greater than $5 \times 10^{-11}$ Torr.

\section{Band structure calculations}

First-principles electronic structure calculations on $\mathrm{TiB}_{2}$ were carried out with the projector augmented wave method $[39,40]$ as implemented in the Vienna ab initio Simulation Package [41]. The generalized gradient approximation of the Perdew-Burke-Ernzerhof formula [42] was adopted for the exchange-correlation functional. The kinetic energy cutoff of the plane-wave basis was set to be $420 \mathrm{eV}$. A $20 \times 20 \times 20$ $k$-point mesh was utilized for the BZ sampling. The FSs were investigated by adopting the maximally localized Wannier function method [43]. The SOC effect was taken into account in all of the above calculations.

[1] C. K. Chiu, J. C. Y. Teo, A. P. Schnyder, and S. Ryu, Classification of Topological Quantum Matter with Symmetries, Rev. Mod. Phys. 88, 035005 (2016).

[2] A. A. Burkov, M. D. Hook, and L. Balents, Topological Nodal Semimetals, Phys. Rev. B 84, 235126 (2011).

[3] Z. K. Liu, B. Zhou, Y. Zhang, Z. J. Wang, H. M. Weng, D. Prabhakaran, S. K. Mo, Z. X. Shen, Z. Fang, X. Dai, Z. Hussain, and Y. L. Chen, Discovery of a Three-Dimensional Topological Dirac Semimetal, $\mathrm{Na}_{3} \mathrm{Bi}$, Science 343, 864 (2014).

[4] S. Borisenko, Q. Gibson, D. Evtushinsky, V. Zabolotnyy, B. Büchner, and R. J. Cava, Experimental Realization of a Three-Dimensional Dirac Semimetal, Phys. Rev. Lett. 113, 027603 (2014).

[5] H. M. Weng, C. Fang, Z. Fang, B. A. Bernevig, and X. Dai, Weyl Semimetal Phase in Noncentrosymmetric TransitionMetal Monophosphides, Phys. Rev. X 5, 011029 (2015).

[6] S. M. Huang, S. Y. Xu, I. Belopolski, C. Lee, G. Chang, B. Wang, N. Alidoust, G. Bian, M. Neupane, C. Zhang, S. Jia, A. Bansil, H. Lin, and M.Z. Hasan, A Weyl Fermion Semimetal with Surface Fermi Arcs in the Transition Metal Monopnictide TaAs Class, Nat. Commun. 6, 7373 (2015).

[7] X. Huang, L. Zhao, Y. Long, P. Wang, D. Chen, Z. Yang, H. Liang, M. Xue, H. Weng, Z. Fang, X. Dai, and G. Chen, Observation of the Chiral-Anomaly-Induced Negative Magnetoresistance in $3 D$ Weyl Semimetal TaAs, Phys. Rev. X 5 , 031023 (2015).

[8] S. Y. Xu et al., Discovery of a Weyl Fermion Semimetal and Topological Fermi Arcs, Science 349, 613 (2015).

[9] B. Q. Lv, H. M. Weng, B. B. Fu, X. P. Wang, H. Miao, J. Ma, P. Richard, X. C. Huang, L. X. Zhao, G. F. Chen, Z. Fang, X. Dai, T. Qian, and H. Ding, Experimental Discovery of Weyl Semimetal TaAs, Phys. Rev. X 5, 031013 (2015).

[10] L. X. Yang, Z. K. Liu, Y. Sun, H. Peng, H. F. Yang, T. Zhang, B. Zhou, Y. Zhang, Y. F. Guo, M. Rahn, D. Prabhakaran, Z. Hussain, S.-K. Mo, C. Felser, B. Yan, and Y. L. Chen, Weyl Semimetal Phase in the Non-Centrosymmetric Compound TaAs, Nat. Phys. 11, 728 (2015). 
[11] S. Y. Xu et al., Discovery of a Weyl Fermion State with Fermi Arcs in Niobium Arsenide, Nat. Phys. 11, 748 (2015).

[12] N. Xu, H. M. Weng, B. Q. Lv, C. E. Matt, J. Park, F. Bisti, V. N. Strocov, D. Gawryluk, E. Pomjakushina, K. Conder, N. C. Plumb, M. Radovic, G. Autès, O. V. Yazyev, Z. Fang, X. Dai, T. Qian, J. Mesot, H. Ding, and M. Shi, Observation of Weyl Nodes and Fermi Arcs in Tantalum Phosphide, Nat. Commun. 7, 11006 (2016).

[13] Z. Zhu, G. W. Winkler, Q. S. Wu, J. Li, and A. A. Soluyanov, Triple Point Topological Metals, Phys. Rev. X 6, 031003 (2016).

[14] B. Q. Lv, Z. L. Feng, Q. N. Xu, X. Cao, J. Z. Ma, L. Y. Kong, P. Richard, Y. B. Huang, V. N. Strocov, C. Fang, H. M. Wang, Y. G. Shi, T. Qian, and H. Ding, Observation of Three-Component Fermions in the Topological Semimetal Molybdenum Phosphide, Nature (London) 546, 627 (2017).

[15] C. Fang, Y. Chen, H. Y. Kee, and L. Fu, Topological Nodal Line Semimetals with and without Spin-Orbital Coupling, Phys. Rev. B 92, 081201 (2015).

[16] T. Bzdušek, Q. Wu, A. Rüegg, M. Sigrist, and A. A. Soluyanov, Nodal-Chain Metals, Nature (London) 538, 75 (2016).

[17] Z. Yan, R. Bi, H. Shen, L. Lu, S. C. Zhang, and Z. Wang, Nodal-Link Semimetals, Phys. Rev. B 96, 041103(R) (2017).

[18] C. Fang, H. M. Weng, X. Dai, and Z. Fang, Topological Nodal Line Semimetals, Chin. Phys. B 25, 117106 (2016).

[19] R. Yu, Z. Fang, X. Dai, and H. Weng, Topological Nodal Line Semimetals Predicted from First-Principles Calculations, Front. Phys. 12, 127202 (2017).

[20] L. M. Schoop, M. N. Ali, C. Straßer, A. Topp, A. Varykhalov, D. Marchenko, V. Duppel, S. S. P. Parkin, B. V. Lotsch, and C. R. Ast, Dirac Cone Protected by Non-Symmorphic Symmetry and Three-Dimensional Dirac Line Node in ZrSiS, Nat. Commun. 7, 11696 (2016).

[21] Q. Xu, R. Yu, Z. Fang, X. Dai, and H. Weng, Topological Nodal Line Semimetals in the $\mathrm{CaP}_{3}$ Family of Materials, Phys. Rev. B 95, 045136 (2017).

[22] X.-B. Wang, X.-M. Ma, E. Emmanouilidou, B. Shen, C.-H. Hsu, C.-S. Zhou, Y. Zuo, R.-R. Song, S.-Y. Xu, G. Wang, L. Huang, N. Ni, and C. Liu, Topological Surface Electronic States in Candidate Nodal-Line Semimetal CaAgAs, Phys. Rev. B 96, 161112(R) (2017).

[23] G. Bian et al., Topological Nodal-Line Fermions in SpinOrbit Metal PbTaSe 2 , Nat. Commun. 7, 10556 (2016).

[24] D. Takane, Z. Wang, S. Souma, K. Nakayama, C. X. Trang, T. Sato, T. Takahashi, and Y. Ando, Dirac-Node Arc in the Topological Line-Node Semimetal HfSiS, Phys. Rev. B 94, 121108(R) (2016).

[25] R. Lou, J.-Z. Ma, Q.-N. Xu, B.-B. Fu, L.-Y. Kong, Y.-G. Shi, P. Richard, H.-M. Weng, Z. Fang, S.-S. Sun, Q. Wang, H.-C. Lei, T. Qian, H. Ding, and S.-C. Wang, Emergence of Topological Bands on the Surface of ZrSnTe Crystal, Phys. Rev. B 93, 241104(R) (2016).

[26] M. M. Hosen, K. Dimitri, I. Belopolski, P. Maldonado, R. Sankar, N. Dhakal, G. Dhakal, T. Cole, P. M. Oppeneer, D. Kaczorowski, F. Chou, M. Z. Hasan, T. Durakiewicz, and M. Neupane, Tunability of the Topological Nodal-Line Semimetal Phase in ZrSiX-Type Materials $(X=S$, Se, Te), Phys. Rev. B 95, 161101(R) (2017).
[27] X. Zhang, Z. M. Yu, X. L. Sheng, H. Y. Yang, and S. A. Yang, Coexistence of Four-Band Nodal Rings and Triply Degenerate Nodal Points in Centrosymmetric Metal Diborides, Phys. Rev. B 95, 235116 (2017).

[28] X. Feng, C. Yue, Z. Song, Q. Wu, and B. Wen, Topological Dirac Nodal-Net Fermions in $\mathrm{AlB}_{2}$-Type $\mathrm{TiB}_{2}$ and $\mathrm{ZrB}_{2}$, Phys. Rev. Mater. 2, 014202 (2018).

[29] B. Post, F. W. Glaser, and D. Moskowitz, Transition Metal Diborides, Acta Metall. 2, 20 (1954).

[30] B. Xia, P. Ren, A. Sulaev, P. Liu, S.-Q. Shen, and L. Wang, Indications of Surface-Dominated Transport in Single Crystalline Nanoflake Devices of Topological Insulator $\mathrm{Bi}_{1.5} \mathrm{Sb}_{0.5} \mathrm{Te}_{1.8} \mathrm{Se}_{1.2}$, Phys. Rev. B 87, 085442 (2013).

[31] J. Xiong, S. K. Kushwaha, T. Liang, J. W. Krizan, M. Hirschberger, W. Wang, R. J. Cava, and N. P. Ong, Evidence for the Chiral Anomaly in the Dirac Semimetal $\mathrm{Na}_{3} \mathrm{Bi}$, Science 350, 413 (2015).

[32] T. Liang, Q. Gibson, M. N. Ali, M. Liu, R. J. Cava, and N.P. Ong, Ultrahigh Mobility and Giant Magnetoresistance in the Dirac Semimetal $\mathrm{Cd}_{3} \mathrm{As}_{2}$, Nat. Mater. 14, 280 (2015).

[33] J. Hu, Z. Tang, J. Liu, X. Liu, Y. Zhu, D. Graf, K. Myhro, S. Tran, C. N. Lau, J. Wei, and Z. Mao, Evidence of Topological Nodal-Line Fermions in ZrSiSe and ZrSiTe, Phys. Rev. Lett. 117, 016602 (2016).

[34] J. Hu, Z. Tang, J. Liu, Y. Zhu, J. Wei, and Z. Mao, Nearly Massless Dirac Fermions and Strong Zeeman Splitting in the Nodal-Line Semimetal ZrSiS Probed by De Haas-Van Alphen Quantum Oscillations, Phys. Rev. B 96, 045127 (2017).

[35] H. Kumigashira, H.-D. Kim, T. Ito, A. Ashihara, T. Takahashi, T. Suzuki, M. Nishimura, O. Sakai, Y. Kaneta, and H. Harima, High-Resolution Angle-Resolved Photoemission Study of LaSb, Phys. Rev. B 58, 7675 (1998).

[36] See Supplemental Material at http://link.aps.org/ supplemental/10.1103/PhysRevX.8.031044 for bulk band calculations without SOC, EDX, powder XRD, field dependence of the resistivity and Hall resistivity at $T=3 \mathrm{~K}$, slab calculation with 20 unit cells, and the parity products for the occupied states at the time-reversal invariant momenta.

[37] T. Tanaka and Y. Ishizawa, The De Haas-Van Alphen Effect in $\mathrm{TiB}_{2}$, J. Phys. C 13, 6671 (1980).

[38] L. Fu, C. L. Kane, and E. J. Mele, Topological Insulators in Three Dimensions, Phys. Rev. Lett. 98, 106803 (2007).

[39] P.E. Blöchl, Projector Augmented-Wave Method, Phys. Rev. B 50, 17953 (1994).

[40] G. Kresse and D. Joubert, From Ultrasoft Pseudopotentials to the Projector Augmented-Wave Method, Phys. Rev. B 59, 1758 (1999).

[41] G. Kresse and J. Hafner, Ab Initio Molecular Dynamics for Open-Shell Transition Metals, Phys. Rev. B 48, 13115 (1993).

[42] J. P. Perdew, K. Burke, and M. Ernzerhof, Generalized Gradient Approximation Made Simple, Phys. Rev. Lett. 77, 3865 (1996).

[43] I. Souza, N. Marzari, and D. Vanderbilt, Maximally Localized Wannier Functions for Entangled Energy Bands, Phys. Rev. B 65, 035109 (2001). 